\title{
Expressão do comportamento de proteção materna em bovinos: uma revisão
}

\section{Expression of maternal protective behavior in cattle: a review}

\author{
Franciely de Oliveira Costa ${ }^{1,2}$, Tiago da Silva Valente ${ }^{2,3}$, Mateus José Rodrigues Paranhos da Costa ${ }^{2,4}$, Marcia del \\ Campo $^{5^{*}}$ \\ ${ }^{1}$ Programa de Pós-Graduação em Zootecnia, Universidade Estadual Paulista (UNESP), Jaboticabal, SP, Brasil \\ ${ }^{2}$ Grupo de Estudos e Pesquisas em Etologia e Ecologia Animal (ETCO), Universidade Estadual Paulista (UNESP), Jaboticabal, SP, Brasil \\ ${ }^{3}$ Livestock Gentec, University of Alberta (UAlberta), Edmonton, AB, Canadá \\ ${ }^{4}$ Departamento de Zootecnia, Universidade Estadual Paulista (UNESP), Jaboticabal, SP, Brasil \\ ${ }^{5}$ Programa Nacional de Carne y Lana, Instituto Nacional de Investigación Agropecuaria (INIA), Tacuarembó, Uruguay
}

\section{Resumo}

Na cadeia produtiva de bovinos de corte é reconhecida a importância do comportamento materno para a sobrevivência e o desenvolvimento do bezerro recém-nascido. Neste caso, o sucesso da atividade depende de alguns componentes do comportamento materno, dentre eles as relações estabelecidas entre a vaca e o bezerro nas primeiras horas após o parto, a aquisição de imunidade pela ingestão do colostro, o suprimento adequado de leite e a proteção ativa da cria contra eventuais predadores. Este último é conhecido como comportamento de proteção materna, que é caracterizado como a reação das vacas frente à presença de potenciais predadores, incluindo humanos. Após o parto, as vacas podem expressar diferentes comportamentos, desde rejeição do bezerro, que representaria uma falha na formação do vínculo natural entre mãe e filho, até comportamentos de proteção materna muito intensos. Alguns procedimentos de manejo importantes para os bezerros são realizados no primeiro dia após o parto, tais como verificar se houve ingestão do colostro, desinfecção do umbigo, identificação dos bezerros, dentre outros. Se durante esse manejo os vaqueiros são percebidos como potenciais ameaças, uma vaca muito protetora pode expressar comportamentos agressivos em relação a eles na tentativa de proteger sua cria, aumentando o risco de acidentes com ambos, vaqueiro e bezerro. Assim, embora o comportamento de proteção materna seja uma característica desejável, é possível que se torne um risco para a segurança dos trabalhadores. Nesta revisão será abordada a questão do comportamento de proteção materna em bovinos de corte, sua relação com a reatividade ao manejo, e potenciais implicações no desempenho dos bezerros à luz dos resultados das pesquisas realizadas até o momento.

Palavras-chave: Agressividade. Bovinos de corte. Habilidade materna. Reatividade. Temperamento. 


\section{Abstract}

In the beef cattle production chain, the importance of maternal behavior for survival and development of newborn calves is recognized. In this case, the business success depends on some components of maternal behavior, among them the establishment of the relationships between cow and calf during the first hours after calving, immunity acquisition through colostrum ingestion, adequate milk supply, and active protection against predators. The latter is known as maternal protective behavior, which is characterized as cow's reaction towards the presence of potential predators, humans included. After calving, cows can express different behaviors, from rejection of the newborn calf, which would represent a failure in natural bond between mother and calf, up to intense maternal protective behavior. Important procedures are undertaken with calves in the first day after calving, such as verification of colostrum consumption, navel disinfection, calf identification, among others. If during these first handling procedures workers are perceived as potential threats, a protective cow can express aggressive behavior towards them as an attempt to protect its offspring, increasing the risk of accidents for both, handler and calf. Therefore, although maternal protective behavior is a desirable trait, it may become a risk to the workers' safety. This review will address the issue of maternal protective behavior in beef cattle, their relationship with reactivity to handling, and potential implications on calves' performance in light of the results of the research conducted until now.

Keywords: Aggressiveness. Beef cattle. Maternal ability. Reactivity. Temperament.

\section{Introdução}

A fase de cria desempenha um papel fundamental na cadeia produtiva da bovinocultura de corte (Pereira et al., 2015; Souza e Chefer, 2016). Reconhecida pela sua complexidade, envolve todos os processos ligados à reprodução dos bovinos, que se inicia com o manejo de matrizes e reprodutores e é finalizada com a desmama dos bezerros, quando estes atingem por volta de 210 dias de vida (Oliveira et al., 2006).
O pleno sucesso nesta fase do processo produtivo éalcançado quando cada uma das vacas de um plantel desmama um bezerro por ano. No entanto, esta meta é difícil de ser alcançada (Pereira et al., 2015), uma vez que algumas vacas não emprenham a cada ano. Além disso, há perdas de bezerros tanto no período pré-natal quanto no pós-natal, decorrentes de casos de abortos e de mortes de bezerros, respectivamente (Magalhães Silva et al., 2017). Assim, para aproximarse da meta de desmamar um bezerro por vaca por ano, é necessário reduzir perdas, sendo a redução da taxa de mortalidade dos bezerros um importante passo neste sentido. Para tanto, é fundamental assegurar que os bezerros consumam colostro de boa qualidade logo após o nascimento e que o manejo de desinfecção do umbigo seja bem feito (Souza e Chefer, 2016). Além disso, é necessário que as vacas expressem comportamentos afiliativos, que fortalecem a formação do vínculo com seus bezerros, e protejam suas crias em casos de ameaça de potenciais predadores (Grandinson, 2005; Paranhos da Costa et al., 2006; Toledo et al., 2013). Em relação a este último, é possível que algumas vacas apresentem respostas extremas, exibindo comportamentos que variam desde o abandono de seus bezerros até reações de ameaça e/ou ataque aos manejadores, caso estes sejam percebidos como ameaça durante o manejo de identificação e cura do umbigo realizado com os recém-nascidos, aumentando o risco de acidente de trabalho (Buddenberg et al., 1986; Turner et al., 2013).

Vários estudos foram realizados com o objetivo de compreender a expressão do comportamento de proteção materna em diferentes raças bovinas, como Hereford, Angus, Charolais e Red Poll (Buddenberg et al., 1986); Angus, Hereford e seus cruzamentos com Friesian, Jersey, South Devon, Chianina, Simmental (Morris et al., 1994); German Angus e Simmental (Hoppe et al., 2008). Também foram identificados os fatores que influenciam a expressão deste comportamento (Buddenberg et al., 1986; Hoppe et al., 2008; Stěhulová et al., 2013), e sua relação com o temperamento de cada animal (Flörcke et al., 2012; Turner et al., 2013; Pérez-Torres et al., 2014). Complementarmente, alguns estudos trataram este tema com abordagens genéticas, estimando os coeficientes de herdabilidade e de repetibilidade desta característica (Buddenberg 
et al., 1986; Morris et al., 1994; Hoppe et al., 2008; Turner et al., 2013), bem como avaliando os efeitos genéticos do comportamento de proteção materna no desempenho de bezerros de corte (Hoppe et al., 2008; Turner et al., 2013).

No Brasil, o estudo sobre o comportamento de proteção materna está iniciando com foco na variabilidade da característica, sua relação com o temperamento e seus potenciais efeitos no desempenho dos bezerros. Além disso, também está sendo verificada a aplicabilidade da avaliação sistemática desse comportamento durante a rotina de manejo de fazendas comerciais (Costa, 2017). Nesta revisão será abordada a questão do comportamento de proteção materna em bovinos de corte, sua relação com a reatividade ao manejo e potenciais implicações no desempenho dos bezerros à luz dos resultados das pesquisas realizadas até o momento.

\section{Comportamento materno}

De maneira geral, fêmeas da classe dos mamíferos exibem comportamentos complexos durante a gestação, parto e lactação. As alterações no repertório comportamental das fêmeas nesta fase da vida são evidenciadas dias antes do parto, quando podem ser observados isolamento social e aumento na agressividade. Logo após o parto, os primeiros comportamentos apresentados por alguns mamíferos são a limpeza do recémnascido, com o consumo do líquido amniótico e dos envoltórios fetais, a estimulação do recém-nascido e a amamentação (González-Mariscal e Poindron, 2002). Todos esses comportamentos, em conjunto, são essenciais para a formação do vínculo entre a mãe e o filhote, caracterizando uma preferência mútua, afetuosa e emocional que é duradoura, persistente e que pode ter um grande impacto na sobrevivência dos filhotes (Svare, 1981; GonzálezMariscal e Poindron, 2002; Grandinson, 2005).

Os bovinos de corte não são diferentes, sendo que o comportamento de lamber o bezerro logo após o parto é fundamental tanto para a formação do vínculo materno-filial, quanto para estimular a respiração, circulação, micção e defecação do neonato (Lidfors e Jensen, 1988). Além disso, o ato de lamber também pode direcionar o bezerro para a mamada do colostro, permitindo a adequada aquisição de imunidade. Estes estímulos, junto da proteção ativa da cria contra eventuais predadores, são habilidades fundamentais para a sobrevivência e para o crescimento adequado do animal, resultando em filhotes de bom desempenho e saudáveis na desmama (Grandinson, 2005; Toledo et al., 2013). Assim, o sucesso da atividade pecuária está diretamente relacionado a alguns componentes da habilidade materna e das relações estabelecidas entre a vaca e o bezerro nas primeiras horas após o parto (Grandinson, 2005; von Keyserlingk e Weary, 2007; Toledo et al., 2013).

Em sistemas de produção de bovinos de corte, o comportamento materno é ainda mais importante do que na produção leiteira, pois as vacas têm a função de cuidar do bezerro, aproveitando-se da habilidade desta em nutrir e proteger os filhotes (Grandinson, 2005; Sandelin et al., 2005; von Keyserlingk e Weary, 2007). Somados aos cuidados maternos, os manejadores são responsáveis por procedimentos que aumentam a resistência dos bezerros frente às condições ambientais às quais estão expostos, como a assepsia do umbigo e a aplicação de antiparasitário após o nascimento (Schmidek et al., 2009). Além disso, uma implicação prática da atuação dos manejadores é o importante papel que desempenham no controle zootécnico das fazendas, permitindo a correta identificação da díade mãe-filhote e a pesagem dos bezerros, possibilitando uma avaliação sistemática da habilidade materna e do desempenho dos animais.

\section{Comportamento de proteção materna}

A mudança no comportamento social das fêmeas é um aspecto importante do cuidado materno, porém ainda pouco explorado em bovinos. 0 comportamento de proteção materna está relacionado, principalmente, com alterações no nível de agressividade que ocorrem após o parto e que têm papel fundamental na manutenção e evolução das espécies (Svare, 1981; Maestripieri, 1992). A agressividade materna serve para impedir que a prole sofra ataque de coespecíficos e/ou predadores, ou seja, está diretamente relacionada à proteção dos descendentes. Dessa maneira, a 
alteração do comportamento é momento-específica na vida dos animais e, se a prole é removida do convívio materno, a agressão diminui quase que imediatamente, pois a função deste comportamento deixa de existir (Rosenblatt et al., 1994).

Durante a estação de nascimento dos bezerros, o primeiro manejo ocorre com intervalo que varia, normalmente, entre 6 e 24 horas após o parto. Este é um período sensível para a díade, pois compreende a fase de formação do vínculo materno-filial (Enríquez et al., 2011). Este processo de reconhecimento mútuo, no entanto, pode ser perturbado durante a realização de alguns procedimentos de manejo importantes, tais como ajudar os bezerros na ingestão do colostro, realizar a assepsia do umbigo e identificar os bezerros, dentre outros. Se neste momento os manejadores são percebidos como potenciais ameaças aos bezerros, algumas vacas muito protetoras podem expressar comportamentos agressivos na tentativa de proteger a cria, aumentando o risco de acidentes com os vaqueiros, bem como com o próprio bezerro e com outros animais ao redor (Buddenberg et al., 1986). Além dos comportamentos agressivos, algumas vacas podem expressar níveis intermediários de atenção ao bezerro e até rejeição ao recém-nascido, o que representaria uma falha na formação do vínculo natural entre mãe e filho (von Keyserlingk e Weary, 2007).

Além disso, a maneira com que algumas vacas reagem pode interferir na qualidade do manejo, especialmente nos casos em que não há instalações adequadas para a realização do trabalho (Turner e Lawrence, 2007). Por isso, embora a expressão do comportamento de proteção materna seja desejável para as vacas, é possível que determinadas reações comportamentais, associadas à expressão de medo ou de agressividade, tornem-se um elemento de risco para todos os envolvidos no manejo (Phocas et al., 2006; Turner et al., 2013), principalmente em sistemas produtivos onde o contato entre humanos e animais não é frequente (Turner e Lawrence, 2007; Hoppe et al., 2008).

O comportamento de proteção materna aparece na literatura científica com diferentes terminologias como, por exemplo, agressão materna (maternal aggression) (Svare, 1981; Wiener, 2015), temperamento no parto (calving temperament) (Morris et al., 1994), comportamento de proteção materna (maternal protective behavior) (Hoppe et al., 2008; Flörcke et al., 2012; Pérez-Torres et al., 2014) e comportamento de defesa materna (maternal defensiveness behavior) (Turner et al., 2013). Independentemente desta variação na nomenclatura, a avaliação dessa característica comportamental tem sido realizada com base em diferentes metodologias de escores visuais com escalas pré-definidas, variando de cinco a onze notas (Buddenberg et al., 1986; Morris et al., 1994), ou com adaptações destas (Hoppe et al., 2008; Pérez-Torres et al., 2014; Geburt et al., 2015; Costa et al., 2017).

Os escores visuais utilizados na avaliação da proteção materna são compostos, principalmente, por aspectos relacionados à atenção da vaca ao bezerro e à agressividade das vacas em relação à aproximação do manejador (Buddenberg et al., 1986). Esses escores podem variar de total indiferença e baixa agressividade até grande interesse pela prole associado a alto nível de agressividade (Sandelin et al., 2005; Turner e Lawrence, 2007). Esta avaliação geralmente é realizada durante o manejo de identificação do bezerro recém-nascido, aproximadamente 24 horas após o parto (Buddenberg et al., 1986; Morris et al., 1994; Hoppe et al., 2008; Turner et al., 2013; Pérez-Torres et al., 2014). Do ponto de vista prático, a utilização de escores permite avaliar as reações das vacas frente ao distúrbio causado no vínculo vaca-bezerro, identificando diferentes tipos de repostas a esta situação (Hoppe et al., 2008). Sendo assim, considera-se que estes métodos são úteis para serem aplicados em fazendas comerciais, dada a facilidade de mensuração e possibilidade de avaliação de um grande número de animais (Turner e Lawrence, 2007).

Diferentemente da maioria dos estudos apresentados anteriormente, nos quais o comportamento de proteção das vacas foi avaliado, aproximadamente, 24 horas após o parto, Stěhulová et al. (2013) avaliaram a reação das vacas nos dias 4 e 31 pós-parto, e Geburt et al. (2015) no segundo e terceiro dias após o nascimento dos bezerros. Neste último estudo também foi utilizada uma escala visual analógica de $15 \mathrm{~cm}$, onde zero representou uma vaca perfeitamente calma, e 15 , uma vaca que ataca vigorosamente o manejador (Geburt et al., 
2015). Esse método permite que os avaliadores elaborem seus próprios descritores relacionados ao comportamento do animal, caracterizando uma medida mais abrangente, porém com abordagem menos convencional (Turner e Lawrence, 2007). 0 comportamento de proteção materna também foi avaliado por meio de teste a campo, no qual um carro se aproximou da díade gradualmente em círculo, com movimentos que simulavam a aproximação de um predador (Flörcke et al., 2012).

Muitas pesquisas têm focado nas respostas comportamentais dos animais em situações específicas e padronizadas, principalmente aquelas diretamente relacionadas à produtividade da fazenda, segurança dos trabalhadores e bem-estar dos animais, considerando a variabilidade individual como o temperamento (Turner et al., 2011). Nesse contexto, o comportamento de proteção materna poderia ser denominado como temperamento materno, uma vez que indica a reação da vaca ao manejo do bezerro neonato. Independentemente da nomenclatura, ele pode ser considerado um aspecto do temperamento da vaca, que ocorre em um estado fisiológico e em um momento específico da vida do animal.

\section{Fatores que influenciam a proteção materna e seu efeito no desempenho do bezerro}

A expressão do comportamento de proteção materna é alterada com as sucessivas experiências reprodutivas e com o amadurecimento fisiológico de cada animal, de maneira que fêmeas com maior idade ou número de lactações apresentam reações mais intensas de proteção (Buddenberg et al., 1986; Paranhos da Costa et al., 2004; Hoppe et al., 2008). Além disso, vacas primíparas expressam, com frequência, comportamentos diferentes dos esperados como, por exemplo, passar menos tempo lambendo seus bezerros e com menos intensidade do que as multíparas (Le Neindre e D'Hour, 1989). Essas diferenças talvez expliquem a maior intensidade na expressão de comportamentos de proteção materna nos partos subsequentes, sugerindo, também, que as vacas não se habituam aos procedimentos de manejo realizados com os bezerros recém-nascidos (Hoppe et al., 2008).
Outras características que também podem influenciar a expressão de proteção materna são a condição corporal e o estado de saúde das vacas após o parto. De acordo com Stěhulová et al. (2013), fêmeas com melhores condições corporais se mostraram mais protetoras em relação a prole. Além disso, estes autores indicaram que bezerros machos receberam maior proteção de suas mães quando comparados com as fêmeas. No entanto, Buddenberg et al. (1986) não encontraram efeito significativo do sexo do bezerro no comportamento de proteção materna. Desta maneira, estudos adicionais precisam ser realizados com o objetivo de entender melhor as relações entre o sexo do bezerro e o investimento parental, avaliado por meio das reações de proteção materna.

A reação das vacas também pode variar de acordo com o peso de nascimento dos bezerros. Foram observados, porém, resultados divergentes nos estudos realizados por Sandelin et al. (2005) e Stěhulová et al. (2013), sendo que no primeiro os autores observaram que bezerros com melhores condições corporais foram mais protegidos pelas vacas, e no segundo, animais mais leves receberam maior proteção de suas mães, bem como amamentação mais frequente. 0 peso na desmama e o ganho de peso médio diário do bezerro (do nascimento à desmama) não foram influenciados pelo comportamento de proteção materna (Hoppe et al., 2008; Turner et al., 2013). No entanto, o desempenho pode ser afetado pela raça, sexo do bezerro e número de lactações da vaca (Hoppe et al., 2008).

\section{Aspectos genéticos do comportamento de proteção materna}

É amplamente reconhecido que os aspectos comportamentais são influenciados por fatores tanto genéticos quanto ambientais (Mormède, 2005; Jensen et al., 2008), os quais interagem para a modulação do repertório comportamental ao longo da vida dos animais. As situações ou desafios enfrentados (Schwartzkopf-Genswein et al., 1997), o tipo de sistema produtivo (Titto et al., 2010), as estratégias de manejo empregadas (Cooke et al., 2009) e a maneira com que são realizadas as 
interações homem-animal (Boissy e Bouissou, 1988; Becker e Lobato, 1997) são fatores ambientais importantes na modificação do comportamento dos animais de produção.

Da mesma forma, estudos focados na análise genética dos comportamentos de proteção materna também foram desenvolvidos, buscando estimar os coeficientes de herdabilidade e de repetibilidade desta característica (Buddenberg et al., 1986; Morris et al., 1994; Hoppe et al., 2008). As estimativas de herdabilidade para o comportamento de proteção materna variam de baixa a moderada magnitude para diferentes raças (Tabela 1). Os valores reportados indicam que há uma divergência na relação entre o efeito genético aditivo e o efeito ambiental na expressão dessa característica em diferentes raças. No entanto, alguns autores indicaram que é possível aplicar seleção direta para promover o melhoramento genético do comportamento de proteção materna, reduzindo a agressividade das vacas ao longo das gerações (Turner e Lawrence, 2007).

Tabela 1 - Estimativas de herdabilidade (h2) e de repetibilidade para o comportamento de proteção materna

\begin{tabular}{|c|c|c|c|}
\hline Raças & $\begin{array}{c}\text { Herdabilidade } \\
\text { 土 DP }\end{array}$ & $\begin{array}{c}\text { Repetibilidade } \\
\text { × DP }\end{array}$ & Referências \\
\hline $\begin{array}{l}\text { Hereford, Angus, } \\
\text { Charolais e Red } \\
\text { Poll }\end{array}$ & $0,06 \pm 0,01$ & $0,09 \pm 0,02$ & $\begin{array}{l}\text { Buddenberg } \\
\text { et al., } 1986\end{array}$ \\
\hline $\begin{array}{l}\text { Angus, Hereford } \\
\text { e cruzamentos }\end{array}$ & $0,09 \pm 0,03$ & $0,20 \pm 0,02$ & $\begin{array}{l}\text { Morris et al., } \\
1994\end{array}$ \\
\hline Limousin & $0,36 \pm 0,06$ & - & $\begin{array}{l}\text { Phocas et al., } \\
2006\end{array}$ \\
\hline Angus & $0,14 \pm 0,08$ & $0,24 \pm 0,04$ & $\begin{array}{l}\text { Hoppe et al., } \\
2008\end{array}$ \\
\hline Simmental & $0,42 \pm 0,05$ & $0,42 \pm 0,05$ & $\begin{array}{l}\text { Hoppe et al., } \\
2008\end{array}$ \\
\hline
\end{tabular}

Outro aspecto importante da proteção materna é a consistência da reação de cada vaca ao longo de sucessivas lactações. Assim, alguns autores estimaram a repetibilidade desta característica (Tabela 1), reportando valores que variam de 0,09 $\pm 0,02$ (Buddenberg et al., 1986) a 0,42 $\pm 0,05$
(Hoppe et al., 2008). A baixa repetibilidade mostra que existe pouca semelhança entre os escores de proteção materna em consecutivas lactações do mesmo animal, reforçando a importância dos efeitos ambientais na sua expressão (Buddenberg et al., 1986). Além disso, como discutido anteriormente, existe o efeito da idade e das experiências prévias vivenciadas por cada fêmea durante o manejo do bezerro recém-nascido (Hoppe et al., 2008).

Apesar de alguns autores reportarem estimativas de herdabilidade para o comportamento de proteção materna, ainda são necessários mais estudos com abordagens de genética quantitativa e molecular em outras raças, principalmente em bovinos zebuínos. Além disso, é importante estudar as correlações genéticas e fenotípicas entre o comportamento de proteção materna e outras características de interesseeconômico como, por exemplo, a habilidade materna, que é tradicionalmente avaliada pelo desempenho dos bezerros entre o nascimento e a desmama. A partir dessas informações será possível estabelecer estratégias de manejo e de descarte de vacas agressivas, ou seja, aquelas que aumentam o risco de acidentes a todos os envolvidos no manejo de bezerros recém-nascidos.

De acordo com Turner e Lawrence (2007), selecionar animais intermediários para características de medo e cuidado materno, ou incluir seleção contra agressividade em programas de melhoramento, pode promover maior segurança aos trabalhadores. Cabe ressaltar que os efeitos ambientais, como o estilo de interação entre os manejadores e os animais, podem aumentar a expressão do fenótipo indesejável (Buddenberg et al., 1986), aumentando o medo e, consequentemente, a tensão durante o manejo dos bezerros.

\section{Temperamento}

o temperamento é caracterizado pelas diferenças individuais no comportamento, que são repetidas ao longo do tempo e em distintas situações, abrangendo aspectos como agressividade, evitação à novidade, capacidade de assumir riscos, exploração e sociabilidade (Réale et al., 2007). Diante da complexidade desta característica e considerando que o temperamento 
é influenciado por fatores genéticos, ambientais e pela interação entre eles (Fordyce e Goddard, 1984), tem-se utilizado definições operacionais com a finalidade de superar as limitações para avaliá-lo. Assim, para animais de produção, o temperamento tem sido definido como o conjunto de comportamentos dos animais em relação ao contato com humanos durante o manejo (Burrow, 1997), geralmente atribuído ao medo (Fordyce et al., 1982).

A maioria das pesquisas utiliza escores visuais pré-definidos para caracterizar o temperamento dos animais de produção, avaliando o grau de perturbação de cada indivíduo quando este é submetido a uma determinada situação de manejo como, por exemplo, durante a pesagem (Fordyce et al., 1982; Sant'Anna et al., 2013; Haskell et al., 2014). Para isso, são utilizados escores avaliando a movimentação corporal, frequência respiratória, coice, vocalização e posição corporal, com os maiores escores indicando respostas mais intensas e caracterizando animais mais reativos (Fordyce e Goddard, 1984). Um dos métodos mais tradicionais é o escore de reatividade no tronco de contenção (crush score), com notas que variam de 1 (animal muito calmo) a 5 (animal muito excitável) (Sant'Anna et al., 2013; Rueda et al., 2015). Outro método muito utilizado é o teste de velocidade de fuga (também conhecido como velocidade de saída ou flight speed), o qual registra, com a ajuda de cronômetros ou fotocélulas, o tempo que cada animal demora para percorrer uma distância conhecida imediatamente após a saída do tronco de contenção ou da balança. A premissa deste método consiste em caracterizar os animais com maiores velocidades de fuga (ou menores tempos) como mais reativos (Burrow et al., 1988; Petherick et al., 2002; Valente et al., 2016).

Segundo Grandin (1997), animais de temperamento excitável (reativos) são mais suscetíveis ao estresse durante as rotinas de manejo. Para Paranhos da Costa (2000) e Haskell et al. (2014), esta é uma característica funcional, de valor econômico e importante para a produção animal, uma vez que existem evidências da relação direta entre alta reatividade, susceptibilidade ao estresse e aumento nas despesas do sistema produtivo. Assim, o melhoramento genético do temperamento pode resultar na diminuição dos custos (Groen et al., 1997) e em melhorias nas condições de bemestar de humanos e de animais nas fazendas.

De acordo com a própria definição, o temperamento é uma característica consistente ao longo do tempo e correlacionada a outros aspectos comportamentais do indivíduo, que são expressos em diferentes contextos (Réale et al., 2007; Turner et al., 2011). Assim, uma questão que tem chamado atenção de técnicos e pecuaristas é o impacto que a seleção de animais menos reativos pode ter na expressão de outros comportamentos importantes para o sistema produtivo, como o comportamento de proteção materna em vacas de corte (Turner e Lawrence, 2007). Por isso, é preciso compreender as possíveis relações genéticas e fenotípicas existentes entre as reações expressadas pelas vacas nestes diferentes contextos (manejo no curral e proteção da cria).

\section{Relação entre a reatividade ao manejo e o comportamento de proteção materna}

Phocas et al. (2006) e Turner et al. (2013) consideraram a importância de se estudar a relação entre a reatividade ao manejo (geralmente avaliada no curral) e os comportamentos de cuidado e proteção materna. Phocas et al. (2006), avaliado as consequências potenciais da seleção de bovinos da raça Limousin para menor reatividade, observaram melhor desempenho reprodutivo das novilhas, principalmente em função da maior habilidade materna e maior produção de leite. Por outro lado, no estudo realizado por Turner et al. (2013) foi observado que vacas mais reativas no tronco de contenção se alimentaram por períodos mais longos logo após o parto, mas que este comportamento não influenciou o tempo despendido pelas vacas nos cuidados (cheirar, lamber e amamentar) com seus bezerros recém-nascidos.

Neste mesmo estudo foi avaliada a relação entre a reatividade ao manejo e o comportamento de proteção materna de vacas provenientes de duas fazendas (Turner et al., 2013). Para as vacas de uma das fazendas não foi observada qualquer associação entre as três medidas de reatividade utilizadas (escore de tronco, velocidade de saída e escore de isolamento) e o comportamento de 
proteção materna. Já na outra fazenda, as vacas que apresentaram notas mais altas para o escore de tronco (movimentos vigorosos e frequentes no tronco de contenção) também receberam maiores escores de proteção materna (vacas mais protetoras e com movimentos vigorosos) quando seus bezerros foram manejados (Turner et al., 2013). A divergência entre os resultados obtidos nos dois rebanhos mostra a necessidade de que mais estudos sejam realizados a fim de compreender as possíveis relações entre a reatividade ao manejo e o comportamento de proteção materna e seus potenciais impactos na seleção de animais.

\section{Conclusão}

O comportamento de proteção materna tem sido avaliado com o intuito de identificar as diferenças individuais entre vacas do mesmo rebanho e entender as relações genéticas e fenotípicas dessa característica com aspectos produtivos. Com base nas informações apresentadas nesta revisão, ressaltamos a relevância desse tema para a produção de bovinos de corte, uma vez que esta característica pode estar relacionada à habilidade materna, ao desenvolvimento do bezerro e à segurança dos trabalhadores, resultando em melhores condições de bem-estar para todos, humanos e animais, que convivem no ambiente das fazendas de produção de bovinos de corte.

Entretanto, como evidenciado nesta revisão, ainda há questões não respondidas, além de resultados potencialmente conflitantes. São necessários, portanto, mais estudos que avaliem o comportamento de proteção materna em bovinos de corte considerando diferentes raças, principalmente zebuínos, e entre populações mantidas em diferentes contextos. Em relação aos resultados conflitantes, é preciso que mais pesquisas avaliem a possível relação entre a reatividade ao manejo e o comportamento de proteção materna. A continuidade de estudos sobre este tema tornará possível definir estratégias de seleção e de manejo dos bovinos de corte que permitam reduzir a reatividade dos animais durante as rotinas de manejo, sem colocar em risco a habilidade materna das vacas.

\section{Referências}

Becker BG, Lobato JFP. Effect of gentle handling on the reactivity of zebu crossed calves to humans. Appl Anim Behav Sci. 1997;53(3):219-24.

Boissy A, Bouissou MF. Effects of early handling on heifers' subsequent reactivity to humans and to unfamiliar situations. Appl Anim Behav Sci. 1988;20:259-73.

Buddenberg BJ, Brown CJ, Johnson ZB, Honea RS. Maternal behavior of beef cows at parturition. J Anim Sci. 1986;62(1):42-6.

Burrow HM, Seifert GW, Corbet NJ. A new technique for measuring temperament in cattle. Proc Aust Soc Anim Prod. 1988;17:154-7.

Burrow HM. Measurements of temperament and their relationships with performance traits of beef cattle. Anim Breed Abstr. 1997;65(7):477-95.

Cooke RF, Arthington JD, Austin BR, Yelich JV. Effects of acclimation to handling on performance, reproductive, and physiological responses of Brahman-crossbred heifers. J Anim Sci. 2009;87(10):3403-12.

Costa FO. Avaliação do comportamento de proteção materna de vacas Nelore e Hereford e seus potenciais efeitos no ganho de peso dos bezerros [tese]. Jaboticabal: Universidade Estadual Paulista; 2017. 95 p.

Costa FO, Valente TS, Del Campo M, Paranhos da Costa MJR. Factors affecting maternal protective behavior in Nellore cows. 51st Congress of the International Society for Applied Ethology; 7-10 ago 2017; Aarhus, DK. Wageningen: Academic Publishers; 2017.

Enríquez D, Hötzel MJ, Ungerfeld R. Minimising the stress of weaning of beef calves: a review. Acta Vet Scand. 2011;53(1):28.

Flörcke C, Engle TE, Grandin T, Deesing MJ. Individual differences in calf defence patterns in Red Angus beef cows. Appl Anim Behav Sci. 2012;139(3-4):203-8.

Fordyce G, Goddard ME, Seifert GW. The measurement of temperament in cattle and effect of experience and genotype. In: Proceedings of the Australian Society of 
Animal Production. $14^{\circ}$ Biennial Conference; mai 1982; Brisbane, Australia. Sidney: Pergamon Press; 1982. p. 329-32.

Fordyce G, Goddard ME. Maternal influence on the temperament of Bos indicus cross cows. In: Proceedings of the Australian Society of Animal Production. 15 ${ }^{\circ}$ Biennial Conference; fev 1984; Armidale, Australia. Sidney: Pergamon Press; 1984. p. 345-8.

Geburt K, Friedrich M, Piechotta M, Gauly M, von Borstel UK. Validity of physiological biomarkers for maternal behavior in cows - a comparison of beef and dairy cattle. Physiol Behav. 2015;139:361-8.

González-Mariscal G, Poindron P. Parental care in mammals: immediate internal and sensory factors of control. In: Pfaff DW, Arnold AP, Etgen AM, Fahrbach SE, Rubin RT (EE.). Hormones, Brain and Behavior. New York: Academic Press; 2002. p. 215-98.

Grandin T. Assessment of stress during handling and transport. J Anim Sci. 1997;75(1):249-57.

Grandinson K. Genetic background of maternal behaviour and its relation to offspring survival. Livest Prod Sci. 2005;93(1):43-50.

Groen AF, Steine T, Colleau JJ, Pedersen J, Pribyl J, Reinsch $\mathrm{N}$. Economic values in dairy cattle breeding, with special reference to functional traits. Report of an EAAP - working group. Livest Prod Sci. 1997;49(1):1-21.

Haskell MJ, Simm G, Turner SP. Genetic selection for temperament traits in dairy and beef cattle. Front Genet. 2014;5:368.

Hoppe S, Brandt HR, Erhardt G, Gauly M. Maternal protective behavior of German Angus and Simmental beef cattle after parturition and its relation to production traits. Appl Anim Behav Sci. 2008;114(3-4):297-306.

Jensen P, Buitenhuis B, Kjaer J, Zanella A, Mormède P, Pizzari T. Genetics and genomics of animal behaviour and welfare - challenges and possibilities. Appl Anim Behav Sci. 2008;113(4):383-403.
Le Neindre P, D'Hour P. Effects of a postpartum separation on maternal responses in primiparous and multiparous cows. Anim Behav. 1989;37(1):166-8.

Lidfors L, Jensen P. Behaviour of free-ranging beef cows and calves. Appl Anim Behav Sci. 1988;20(3-4):237-47.

Maestripieri D. Functional aspects of maternal aggression in mammals. Can J Zool. 1992;70(6):1069-77.

Magalhães Silva LC, Baldi F, Aboujaoude C, Venturini GC, Albuquerque LG, Paranhos da Costa MJR. Genetic parameter estimates for prenatal and postnatal mortality in Nellore cattle. J Anim Breed Genet. 2017;134(1):27-33.

Mormède P. Molecular genetics of behaviour: research strategies and perspectives for animal production. Livest Prod Sci. 2005;93(1):15-21.

Morris CA, Cullen NG, Kilgour R, Bremner KJ. Some genetic factors affecting temperament in Bos taurus cattle. New Zeal J Agr Res. 1994;37(2):167-75.

Oliveira RL, Barbosa MAAF, Ladeira MM, Silva MMP, Ziviani AC, Bagaldo AR. Nutrição e manejo de bovinos de corte na fase de cria. Rev Bras Saude Prod An. 2006;7(1):57-86.

Paranhos da Costa MJR. Ambiência na produção de bovinos de corte a pasto. XVIII Encontro Anual de Etologia; 2000; Florianópolis, SC. Florianópolis: SBEt; 2000. p. 26-42.

Paranhos da Costa MJR, Toledo LM, Schmidek A. A criação de bezerros de corte: conhecer para melhorar a eficiência. Cultivar Bovinos, Caderno Técnico; 2004(6): 2-7.

Paranhos da Costa MJR, Schmidek A, Toledo LM. Boas Práticas de Manejo: Bezerros ao Nascimento. Jaboticabal: Funep; 2006.

Pereira LC, Mateus RG, Carvalho CME, Mateus RG, Silva DCG, Borges OA. Avaliação da viabilidade para produção de bezerro de corte a partir dos centros de custo. REPAE. 2015;1(2):187-209. 
Pérez-Torres L, Orihuela A, Corro M, Rubio I, Cohen A, Galina CS. Maternal protective behavior of zebu type cattle (Bos indicus) and its association with temperament. J Anim Sci. 2014;92(10):4694-700.

Petherick JC, Holroyd RG, Doogan VJ, Venus BK. Productivity, carcass and meat quality of lot fed Bos indicus cross steers grouped according to temperament. Aust J Exp Agric. 2002;42(4):389-98.

Phocas F, Boivin X, Sapa J, Trillat G, Boissy A, Le Neindre P. Genetic correlations between temperament and breeding traits in Limousin heifers. Anim Sci. 2006;82(6):805-11.

Réale D, Reader SM, Sol D, McDougall PT, Dingemanse NJ. Integrating animal temperament within ecology and evolution. Biol Rev Camb Philos Soc. 2007;82(2):291-318.

Rosenblatt JS, Factor EM, Mayer AD. Relationship between maternal aggression and maternal care in the rat. Aggress Behav. 1994;20(3):243-55.

Rueda PM, Sant'Anna AC, Valente TS, Paranhos da Costa MJR. Impact of the temperament of Nellore cows on the quality of handling and pregnancy rates in fixed-time artificial insemination. Livest Sci. 2015;177:189-95.

Sandelin BA, Brown Jr AH, Johnson ZB, Hornsby JA, Baublits RT, Kutz BR. Postpartum maternal behavior score in six breed groups of beef cattle over twenty-five years. Prof Anim Sci. 2005;21(1):13-6.

Sant'Anna AC, Paranhos da Costa MJR, Baldi F, Albuquerque LG. Genetic variability for temperament indicators of Nellore cattle. J Anim Sci. 2013;91(8):3532-7.

Schmidek A, Durán H, Paranhos da Costa MJR. Boas práticas de manejo: Identificação. Jaboticabal: Funep; 2009. 39 p.

Schwartzkopf-Genswein KS, Stookey JM, Janzen ED, Mckinnon J. Effects of branding on weight gain, antibiotic treatment rates and subsequent handling ease in feedlot cattle. Can J Anim Sci. 1997;77(3):361-7.

Souza DM, Chefer DM. Bovinocultura de corte: Principais manejos durante a fase de cria. Rev Iniciare. 2016;1(1):40-78.
Stěhulová I, Špinka M, Šárová R, Máchová L, Kněz R, Firla P. Maternal behaviour in beef cows is individually consistent and sensitive to cow body condition, calf sex and weight. Appl Anim Behav Sci. 2013;144(3-4):89-97.

Svare BB. Maternal aggression in mammals. In: Gubernick DJ, Klopfer PH (EE.). Parental Care in Mammals. Nova York: Plenum Press; 1981. p. 179-210.

Titto EAL, Titto CG, Gatto EG, Noronha CMS, Mourão GB, Nogueira Filho JCM, et al. Reactivity of Nellore steers in two feedlot housing systems and its relationship with plasmatic cortisol. Livest Sci. 2010;129(1-3):146-50.

Toledo LM, Paranhos da Costa MJR, Schmidek A, Jung J, Ciryllo JNSG, Cromberg VU. The presence of black vultures at the calving sites and its effects on cows' and calves' behaviour immediately following parturition. Animal. 2013;7(3):469-75.

Turner SP, Lawrence AB. Relationship between maternal defensive aggression, fear of handling and other maternal care traits in beef cows. Livest Sci. 2007;106(2-3):182-8.

Turner SP, Gibbons JM, Haskell MJ. Developing and validating measures of temperament in livestock. In: Inoue-Murayama $M$, Kawamura $S$, Weiss A (EE.). From Genes to Animal Behavior. Tokyo: Springer; 2011. p. 201-24.

Turner SP, Jack MC, Lawrence AB. Precalving temperament and maternal defensiveness are independent traits but precalving fear may impact calf growth. J Anim Sci. 2013;91(9):4417-25.

Valente TS, Baldi F, Sant'Anna AC, Albuquerque LG, Paranhos da Costa MJR. Genome-wide association study between single nucleotide polymorphisms and flight speed in Nellore cattle. PLoS One. 2016;11(6):e0156956.

von Keyserlingk MAG, Weary DM. Maternal behavior in cattle. Horm Behav. 2007;52(1):106-13.

Wiener P. Genetics of behaviour in cattle. In: Garrick DJ, Ruvinsky A (EE.). The genetics of cattle. Wallingford: CABI; 2015. p. 234-59. 